Canad. Math. Bull. Vol. 62 (2), 2019 pp. 259-266

http://dx.doi.org/10.4153/CMB-2018-022-9

(C) Canadian Mathematical Society 2018

\title{
A Note About the Strong Maximum Principle on RCD Spaces
}

\author{
Nicola Gigli and Chiara Rigoni
}

Abstract. We give a direct proof of the strong maximum principle on finite dimensional RCD spaces

based on the Laplacian comparison of the squared distance.

\section{Introduction}

In the context of analysis in metric measure spaces, it is by now well understood that a doubling condition and a Poincare inequality are sufficient to derive the basics of elliptic regularity theory. In particular, one can obtain the Harnack inequality for harmonic functions, which in turns implies the strong maximum principle. We refer the reader to [6] for an overview on the topic and a detailed bibliography.

$\operatorname{RCD}^{*}(K, N)$ spaces $([3,16]$, see also $[4,5,11])$ are, for finite $N$, doubling $([27])$ and support a Poincaré inequality ([24]), and thus, in particular, the above applies (see $[16,17]$ for the details). Still, given that, in fact, such spaces are much more regular than general doubling and Poincaré spaces, one might wonder whether there is a simpler proof of the strong maximum principle.

The aim of this short note is to show that out of the several arguments available in the Euclidean space, the one based on the estimates for the Laplacian of the squared distance carries over to such non-smooth context rather easily. We emphasize that such an argument is, with only minor variations, the original one of Hopf, which appeared in [20] (the so called "boundary point lemma" about the sign of external derivative at a maximum point at the boundary, also due to Hopf, appeared later in [21]).

In order to mimic Hopf's proof we need to know that given a closed set $C$, for 'many' points $x \notin C$ there is a unique $y \in C$ minimizing the distance from $x$. In the Euclidean setting this is easy to prove thanks to the strict convexity of balls, but in general metric spaces the same property can fail, even in presence of a (non-Riemannian) curvature-dimension condition; see Remark 2.7. In our situation this can be proved using the existence of optimal transport maps proved in [18]; see Lemma 2.6. After the work on this manuscript finished, we realized that a very similar statement has been obtained with a similar proof; see [12, Theorem 4.7].

Received by the editors February 20, 2018; revised May 2, 2018.

Published electronically July 11, 2018.

This research has been supported by the MIUR SIR-grant 'Nonsmooth Differential Geometry' (RBSI147UG4)

AMS subject classification: $31 \mathrm{E} 05,35 \mathrm{~B} 50$.

Keywords: maximum principle, RCD space. 
We conclude remarking that this result simplifies the proofs of those properties of $\operatorname{RCD}^{*}(K, N)$ spaces that depend on the strong maximum principle such as the splitting theorem $([14,15])$.

\section{Result}

We will consider all metric measure spaces $(X, d, \mathfrak{m})$ to be such that $(X, d)$ is complete and separable, and $\mathfrak{m}$ will be a Radon non-negative measure with $\operatorname{supp}(\mathfrak{m})=X$.

To keep the presentation short, we assume that the reader is familiar with the definition of $\mathrm{RCD}^{*}$ spaces and with calculus on them $($ see $[3,16])$. In this paper, we only recall those definitions and facts that will be used in the course of the proofs. In particular, we will take for granted the notion of $W^{1,2}(\mathrm{X})$ space on the metric measure space $(\mathrm{X}, \mathrm{d}, \mathfrak{m})$ and, for $f \in W^{1,2}(\mathrm{X})$, of the minimal weak upper gradient $|\mathrm{D} f|$. Recall that the minimal weak upper gradient is a local object, i.e.,

$$
|\mathrm{D} f|=|\mathrm{D} g| \quad \mathfrak{m}-\text { a.e. on }\{f=g\} \quad \forall f, g \in W^{1,2}(\mathrm{X}) .
$$

Then the notion of Sobolev space over an open set can be easily given.

Definition 2.1 (Sobolev space on an open subset of X) Let $(\mathrm{X}, \mathrm{d}, \mathfrak{m})$ be a metric measure space and let $\Omega \subset$ X open. Then we define

$$
\begin{aligned}
W_{\text {loc }}^{1,2}(\Omega) & :=\left\{f \in L_{\text {loc }}^{2}(\Omega): \text { for every } x \in \Omega \text { there exists } U \subset \Omega\right. \\
& \text { neighbourhood of } \left.x \text { and there exists } f_{U} \in W_{\text {loc }}^{1,2}(\mathrm{X}) \text { such that } f_{\left.\right|_{U}}=f_{U}\right\} .
\end{aligned}
$$

For $f \in W_{\text {loc }}^{1,2}(\Omega)$, the function $|\mathrm{D} f| \in L_{\text {loc }}^{2}(\Omega)$ is defined as

$$
|\mathrm{D} f|:=\left|\mathrm{D} f_{U}\right| \quad \mathfrak{m}-\text { a.e. on } U,
$$

where $\left|\mathrm{D} f_{U}\right|$ is the minimal weak upper gradient of $f_{U}$ and the locality of this object ensures that $|\mathrm{D} f|$ is well defined.

Then we set

$$
W^{1,2}(\Omega):=\left\{f \in W_{\text {loc }}^{1,2}(\Omega): f,|\mathrm{D} f| \in L^{2}(\Omega)\right\} .
$$

The definition of (sub/super)-harmonic functions can be given in terms of minimizers of the Dirichlet integral (see [6] for a thorough discussion on the topic).

Definition 2.2 (Subharmonic/Superharmonic/Harmonic functions) Let (X, d, $\mathfrak{m})$ be a metric measure space and let $\Omega$ be an open subset in X. We say that $f$ is subharmonic (resp. superharmonic) in $\Omega$ if $f \in W^{1,2}(\Omega)$ and for any $g \in W^{1,2}(\Omega), g \leq 0$ (resp. $g \geq 0$ ) with supp $g \subset \subset \Omega$, it holds that

$$
\frac{1}{2} \int_{\Omega}|\mathrm{D} f|^{2} \mathrm{dm} \leq \frac{1}{2} \int_{\Omega}|\mathrm{D}(f+g)|^{2} \mathrm{dm} .
$$

The function $f$ is harmonic in $\Omega$ if it is both subharmonic and superharmonic. 
On $\operatorname{RCD}(K, \infty)$ spaces, the weak maximum principle can be deduced directly from the definition of subharmonic function and the following property, proved in [3]:

(2.2) Let $(\mathrm{X}, \mathrm{d}, \mathfrak{m})$ be $\operatorname{RCD}(K, \infty), K \in \mathbb{R}$, and let $f \in W^{1,2}(\mathrm{X})$ be such that $|\mathrm{D} f| \in L^{\infty}(\mathrm{X})$. Then there exists $\widetilde{f}=f \mathfrak{m}$-a.e. such that $\operatorname{Lip}(\widetilde{f}) \leq\|\mathrm{D} f \mid\|_{L^{\infty}}$.

We can now easily prove the following theorem.

Theorem 2.3 (Weak Maximum Principle) Let $(\mathrm{X}, \mathrm{d}, \mathfrak{m})$ be an $\operatorname{RCD}(K, \infty)$ space, let $\Omega \subset \mathrm{X}$ be open with finite measure, and let $f \in W^{1,2}(\Omega) \cap C(\bar{\Omega})$ be subharmonic. Then

$$
\sup _{\Omega} f \leq \sup _{\partial \Omega} f
$$

to be interpreted as " $f$ is constant" in the case $\Omega=\mathrm{X}$.

Proof We argue by contradiction. If (2.3) does not hold, regardless of whether $\Omega$ coincides with $\mathrm{X}$ or not, we can find $c<\sup _{\Omega} f$ such that the function

$$
\widetilde{f}:=\min \{c, f\}
$$

agrees with $f$ on $\partial \Omega$. The locality of the differential grants that

$$
|\mathrm{D} \widetilde{f}|=\chi_{\{f<c\}}|\mathrm{D} f|
$$

and from the assumption that $f$ is subharmonic and the fact that $\widetilde{f} \leq f$, we deduce that

which forces

$$
\int_{\Omega}|\mathrm{D} f|^{2} \mathrm{dm} \leq \int_{\Omega}|\mathrm{D} \widetilde{f}|^{2} \mathrm{dm} \stackrel{(2.4)}{=} \int_{\{f<c\} \cap \Omega}|\mathrm{D} f|^{2} \mathrm{dm},
$$

$$
|\mathrm{D} f|=0 \mathfrak{m} \text {-a.e. on }\{f \geq c\} .
$$

Now consider the function $g:=\max \left\{c, \chi_{\Omega} f\right\}$; notice that our assumptions grant that $g \in C(\mathrm{X})$ and that the locality of the differential yields

$$
|\mathrm{D} g|=\chi_{\Omega \cap\{f>c\}}|\mathrm{D} f| \stackrel{(2.5)}{=} 0 .
$$

Hence, property (2.2) gives that $g$ is constant, i.e., $f \leq c$ on $\Omega$. This contradicts our choice of $c$ and gives the conclusion.

We remark that in the finite-dimensional case one could conclude from (2.6) by using the Poincaré inequality in place of property (2.2).

To prove the strong maximum principle, we need to recall few facts. The first is the concept of a measure-valued Laplacian (see [16]), for which we restrict our attention to proper (i.e., closed bounded sets are compact) and infinitesimally Hilbertian (i.e., $W^{1,2}(\mathrm{X})$ is an Hilbert space, see [16]) spaces. Recall that on infinitesimally Hilbertian spaces, given two functions $f, g \in W_{\mathrm{loc}}^{1,2}$ the quantity $\langle\nabla f, \nabla g\rangle \in L_{\text {loc }}^{1}$ is well defined by polarization of $f \mapsto|\mathrm{D} f|^{2}$ (see [16]).

Definition 2.4 (Measure-valued Laplacian) Let $(\mathrm{X}, \mathrm{d}, \mathfrak{m})$ be proper and infinitesimally Hilbertian, let $\Omega \subset \mathrm{X}$ be open, and let $f \in W^{1,2}(\Omega)$. We say that $f$ has a measure-valued Laplacian in $\Omega$, and write $f \in D(\Delta, \Omega)$, provided there exists a Radon 
measure that we denote by $\Delta f_{\left.\right|_{\Omega}}$, such that for every $g: \mathrm{X} \rightarrow$ Lipschitz with compact support contained in $\Omega$, it holds that

$$
\int g \mathrm{~d} \Delta f_{\left.\right|_{\Omega}}=-\int\langle\nabla f, \nabla g\rangle \mathrm{dm} .
$$

If $\Omega=\mathrm{X}$, we write $f \in D(\Delta)$ and $\Delta f$.

Much like in the smooth case, it turns out that being subharmonic is equivalent to having non-negative Laplacian. This topic has been investigated in $[16,17]$. Here we report the proof of this fact, because in [17] the presence of a Poincaré inequality has been assumed, while working on proper infinitesimally Hilbertian spaces allows us to easily remove such assumption.

Theorem 2.5 Let $(\mathrm{X}, \mathrm{d}, \mathfrak{m})$ be a proper infinitesimally Hilbertian space, $\Omega \subset \mathrm{X}$ open, and $f \in W^{1,2}(\Omega)$.

Then $f$ is subharmonic (resp. superharmonic, harmonic) if and only if $f \in D(\Delta, \Omega)$ with $\Delta f_{\left.\right|_{\Omega}} \geq 0$ (resp. $\Delta f_{\left.\right|_{\Omega}} \leq 0$, resp. $\left.\Delta f_{\left.\right|_{\Omega}}=0\right)$.

Proof

Only if: Let $\operatorname{LIP}_{c}(\Omega) \subset W^{1,2}(\Omega)$ be the space of Lipschitz functions with support compact and contained in $\Omega$. For $g \in \operatorname{LIP}_{c}(\Omega)$ non-positive and $\varepsilon>0$ apply (2.1) with $\varepsilon g$ in place of $g$ to deduce

$$
\int_{\Omega}|\mathrm{D}(f+\varepsilon g)|^{2}-|\mathrm{D} f|^{2} \mathrm{~d} \mathfrak{m} \geq 0 .
$$

Dividing by $\varepsilon$ and letting $\varepsilon \downarrow 0$ we conclude

$$
\int_{\Omega}\langle\nabla f, \nabla g\rangle \mathrm{dm} \geq 0
$$

In other words, the linear functional $\operatorname{LIP}_{c}(\Omega) \ni g \mapsto-\int_{\Omega}\langle\nabla f, \nabla g\rangle \mathrm{dm}$ is positive. It is then well known, see e.g., [7, Theorem 7.11.3], that the monotone extension of such a functional to the space of continuous and compactly supported functions on $\Omega$ is uniquely represented by integration with respect to a non-negative measure, which is the claim.

If: Recall from [2] that on general metric measure spaces Lipschitz functions are dense in energy in $W^{1,2}$; since infinitesimally Hilbertian implies uniform convexity of $W^{1,2}$, we see that in our case they are dense in the $W^{1,2}$-norm. Then by truncation and cut-off argument we easily see that

$$
\begin{aligned}
& \left\{g \in \operatorname{LIP}_{c}(\Omega): g \leq 0\right\} \quad \text { is } W^{1,2}-\text { dense in } \\
& \left.\qquad g \in W^{1,2}(\Omega): g \leq 0 \operatorname{supp}(g) \subset \subset \Omega\right\} .
\end{aligned}
$$

Now notice that the convexity of $g \mapsto \frac{1}{2} \int_{\Omega}|\mathrm{D} g|^{2} \mathrm{dm}$ grants that for any $g \in W^{1,2}(\Omega)$ it holds

$$
|\mathrm{D}(f+g)|^{2}-|\mathrm{D} f|^{2} \geq \lim _{\varepsilon \downarrow 0} \frac{|\mathrm{D}(f+\varepsilon g)|^{2}-|\mathrm{D} f|^{2}}{\varepsilon}=2\langle\nabla f, \nabla g\rangle
$$


and thus from the assumption $\Delta f_{\left.\right|_{\Omega}} \geq 0$ we deduce that

$$
\int_{\Omega}|\mathrm{D}(f+g)|^{2}-|\mathrm{D} f|^{2} \mathrm{dm} \geq 0
$$

for every $g \in \operatorname{LIP}_{c}(\Omega)$ non-positive. Taking (2.7) into account, we see that (2.8) also holds for any $g \in W^{1,2}(\Omega)$ non-negative with $\operatorname{supp}(g) \subset \Omega$, which is the thesis.

For $x \in \mathrm{X}$, we write $\mathrm{d}_{x}$ for the function $y \mapsto \mathrm{d}(x, y)$. We shall need the following two properties of the squared distance function valid on $\operatorname{RCD}^{*}(K, N)$ spaces, $N<\infty$ :

$$
\begin{array}{rll}
\mathrm{d}_{x_{0}}^{2} \in W_{\mathrm{loc}}^{1,2}(\mathrm{X}) & \text { and } \quad\left|\mathrm{D}\left(\mathrm{d}_{x_{0}}^{2}\right)\right|^{2}=2 \mathrm{~d}_{x_{0}}^{2} \quad \text { m-a.e. } \\
\mathrm{d}_{x_{0}}^{2} \in D(\Delta) & \text { and } \quad \Delta \mathrm{d}_{x_{0}}^{2}(x) \leq \ell_{K, N}\left(\mathrm{~d}_{x_{0}}\right) \mathfrak{m},
\end{array}
$$

where $\ell_{K, N}:[0,+\infty) \rightarrow[0,+\infty)$ is some continuous function depending only on $K, N$. Property (2.9) can be seen as a consequence of Cheeger's work [10]. Recall that $\mathrm{CD}(K, N)$ spaces are doubling ([27]) and support a 1-2 weak Poincaré inequality ([24]), and notice that, since $\mathrm{X}$ is geodesic, the local Lipschitz constant of $\mathrm{d}_{x}$ is identically 1 . An alternative proof, more tailored to the RCD setting, makes use of the fact that $\mathrm{d}_{x_{0}}^{2} / 2$ is $c$-concave and uses the regularity of $W_{2}$-geodesics; see, for instance, [19] for the details of the argument.

The Laplacian comparison estimate (2.10) is one of the main results in [16]. Notice that in [16] such inequality has been obtained in its sharp form, but for our purposes, the above formulation is sufficient.

Besides these facts, we need the following geometric property of RCD spaces, which we believe is interesting on its own. For the notions of $c$-transform and $c$-superdifferential; see, for instance, $[1,26,28]$.

Lemma 2.6 (a.e. unique projection) Let $K \in \mathbb{R}, N \in[1, \infty),(\mathrm{X}, \mathrm{d}, \mathfrak{m})$ an $\operatorname{RCD}^{*}(K, N)$ space and $C \subset \mathrm{X}$ a closed set. Then for $\mathfrak{m}$-a.e. $x \in \mathrm{X}$, there exists a unique $y \in C$ such that

$$
\mathrm{d}(x, y)=\min _{z \in C} \mathrm{~d}(x, z) .
$$

Proof Existence follows trivially from the fact that $\mathrm{X}$ is proper. For uniqueness, define

$$
\varphi(x):=\inf _{z \in C} \frac{\mathrm{d}^{2}(x, z)}{2}=\psi^{c}(x) \quad \text { where, } \quad \psi(y):= \begin{cases}0 & \text { if } y \in C, \\ -\infty & \text { if } y \in \mathrm{X} \backslash C .\end{cases}
$$

Since $\varphi^{c}=\psi^{c c} \geq \psi$, if $x \in \mathrm{X}$ and $y \in C$ are such that (2.11) holds, we have

$$
\varphi(x)+\varphi^{c}(y) \geq \varphi(x)+\psi(y) \stackrel{(2.11)}{=} \frac{\mathrm{d}^{2}(x, y)}{2},
$$

i.e., $y \in \partial^{c} \varphi(x)$. Conclude recalling that since $\varphi$ is $c$-concave and real valued, [18, Theorem 3.4] grants that for $\mathfrak{m}$-a.e. $x$ there exists a unique $y \in \partial^{c} \varphi(x)$.

Remark 2.7 The simple proof of this lemma relies on quite delicate properties of RCD spaces; indeed, notice that the conclusion can fail on the more general $\operatorname{CD}(K, N)$ spaces. Consider, for instance, $\mathbb{R}^{2}$ equipped with the distance coming from the $L^{\infty}$ 
norm and the Lebesgue measure $\mathcal{L}^{2}$. This is a $\operatorname{CD}(0,2)$ space, as shown in the last theorem in [28]. Then pick $C:=\left\{\left(z_{1}, z_{2}\right): z_{1} \geq 0\right\}$ and notice that for every $\left(x_{1}, x_{2}\right) \in \mathbb{R}^{2}$ with $x_{1}<0$, there are uncountably many minimizers in (2.11).

What makes the proof work in the RCD case is the validity of the result in [18], which uses some forms of non-branching and lower Ricci bounds to deduce existence of optimal maps. This kind of argument appeared first in [13] (albeit the main idea of the proof was independently discovered by various authors; see, for instance, [8] and the references therein) and since then the topic has been pushed quite far: to date, the most general results are those recently obtained by Kell in [22], which in particular cover the case of essential non-branching (see [25]) MCP spaces (see [23, 27]), previously considered by Cavalletti and Mondino in [9].

We can now prove the main result of this note.

Theorem 2.8 (Strong Maximum Principle) Let $K \in \mathbb{R}, N \in[1, \infty)$ and let $(\mathrm{X}, \mathrm{d}, \mathfrak{m}$ ) be an $\operatorname{RCD}^{*}(K, N)$ space. Let $\Omega \subset \mathrm{X}$ be open and connected and let $f \in W^{1,2}(\Omega) \cap$ $C(\bar{\Omega})$ be subharmonic and such that for some $\bar{x} \in \Omega$, it holds that $f(\bar{x})=\max _{\bar{\Omega}} f$. Then $f$ is constant.

Proof Put $m:=\sup _{\Omega} f, C:=\{x \in \bar{\Omega}: f(x)=m\}$ and define

$$
\Omega^{\prime}:=\{x \in \Omega \backslash C: \mathrm{d}(x, C)<\mathrm{d}(x, \partial \Omega)\} .
$$

By assumption, we know that $C \cap \Omega \neq \varnothing$ and that $\Omega$ is connected, thus, since $C$ is closed, either $C \supset \Omega$, in which case we are done, or $\partial C \cap \Omega \neq \varnothing$, in which case $\Omega^{\prime} \neq \varnothing$. We now show that such second case cannot occur, thus concluding the proof.

Assume by contradiction that $\Omega^{\prime} \neq \varnothing$; notice that $\Omega^{\prime}$ is open and thus $\mathfrak{m}\left(\Omega^{\prime}\right)>0$. Hence, by Lemma 2.6 we can find $x \in \Omega^{\prime}$ and $y \in C$ such that (2.11) holds. Notice that the definition of $\Omega^{\prime}$ grants that $y \in \Omega$, put $r:=\mathrm{d}(x, y)$ and define

$$
h(z):=e^{-A d^{2}(z, x)}-e^{-A r^{2}},
$$

where $A \gg 1$ will be fixed later. By the chain rule for the measure-valued Laplacian (see [16]), we have that $h \in D(\Delta)$ with

$$
\Delta h=A^{2} e^{-A d_{x}^{2}}\left|\operatorname{Dd}_{x}^{2}\right|^{2} \mathfrak{m}-A e^{-A d_{x}^{2}} \Delta \mathrm{d}_{x}^{2} \stackrel{(2.9),(2.10)}{\geq} 2 e^{-A d_{x}^{2}}\left(A^{2} d_{x}^{2}-A \ell_{K, N}\left(\mathrm{~d}_{x}\right)\right) \mathfrak{m} .
$$

Hence we can, and will, choose $A$ so big that $\left.\Delta h\right|_{B_{r / 2}(y)} \geq 0$. Now let $r^{\prime}<r / 2$ be such that $B_{r^{\prime}}(y) \subset \Omega$ and notice that for every $\varepsilon \stackrel{B_{r} / 2}{>} 0$ the function $f_{\varepsilon}:=f+\varepsilon h$ is subharmonic in $B_{r^{\prime}}(y)$, and thus according to Theorem 2.3, we have

$$
f_{\varepsilon}(y) \leq \sup _{\partial B_{r^{\prime}}(y)} f_{\varepsilon}, \quad \forall \varepsilon>0 .
$$

Since $\{h<0\}=\mathrm{X} \backslash \bar{B}_{r}(x)$ and $h(y)=0$, we have

$$
f_{\varepsilon}(y)>f_{\varepsilon}(z) \quad \forall z \in \partial B_{r^{\prime}}(y) \backslash \bar{B}_{r}(x), \quad \forall \varepsilon>0 .
$$

On the other hand, $\partial B_{r^{\prime}}(y) \cap \bar{B}_{r}(x)$ is a compact set contained in $\Omega \backslash C$, hence by continuity and the definition of $C$, we have

$$
f(y)>\sup _{\partial B_{r^{\prime}}(y) \cap \bar{B}_{r}(x)} f,
$$


and thus for $\varepsilon>0$ sufficiently small, we also have

$$
f_{\varepsilon}(y)>\sup _{\partial B_{r^{\prime}}(y) \cap \bar{B}_{r}(x)} f_{\varepsilon} .
$$

This inequality, (2.13), and the continuity of $f_{\varepsilon}$ contradict (2.12); the conclusion follows.

Remark 2.9 The proof uses the weak maximum principle, the Laplacian comparison of the distance, its linearity, and Lemma 2.6 only. Since the Laplacian comparison for the distance holds in the more general class of infinitesimally strictly convex MCP spaces (see [16]), taking Remark 2.7 into account we see that the strong maximum principle holds in the class of essentially non-branching and infinitesimally Hilbertian MCP spaces.

Acknowledgments We thank M. Kell and A. Mondino for useful comments on a preliminary version of this paper and M. Kell also for his help with the translation of Hopf's work [20]. We also wish to thank the referee for the careful reading and the suggestions given.

\section{References}

[1] L. Ambrosio and N. Gigli, A user's guide to optimal transport. In: Modelling and optimisation of flows on networks, Lecture Notes in Mathematics, 2062, Fond. CIME/CIME Found. Subser., Springer, Heidelberg, 2013, pp. 1-155. http://dx.doi.org/10.1007/978-3-642-32160-3_1

[2] L. Ambrosio, N. Gigli, and G. Savaré, Calculus and heat flow in metric measure spaces and applications to spaces with Ricci bounds from below. Invent. Math. 195(2014), 289-391. http://dx.doi.org/10.1007/s00222-013-0456-1

[3] $\longrightarrow$ Metric measure spaces with Riemannian Ricci curvature bounded from below. Duke Math. J. 163(2014), no. 7, 1405-1490. http://dx.doi.org/10.1215/00127094-2681605

[4] Bakry-Émery curvature-dimension condition and Riemannian Ricci curvature bounds. Ann. Probab. 43(2015), 339-404. http://dx.doi.org/10.1214/14-AOP907

[5] L. Ambrosio, A. Mondino, and G. Savaré, Nonlinear diffusion equations and curvature conditions in metric measure spaces. 2015. arxiv:1509.07273 http://dx.doi.org/10.1007/s12220-014-9537-7

[6] A. Björn and J. Björn, Nonlinear potential theory on metric spaces. EMS Tracts in Mathematics, 17, European Mathematical Society (EMS), Zürich, 2011. http://dx.doi.org/10.4171/099

[7] V. Bogachev, Measure theory. Vol. I, II, Springer-Verlag, Berlin, 2007. http://dx.doi.org/10.1007/978-3-540-34514-5

[8] F. Cavalletti, An overview of $L^{1}$ optimal transportation on metric measure spaces. Partial Differ. Equ. Meas. Theory, De Gruyter Open, Warsaw, 2017.

[9] F. Cavalletti and A. Mondino, Optimal maps in essentially non-branching spaces. Commun. Contemp. Math. 19(2017), no. 6, 1750007. http://dx.doi.org/10.1142/S0219199717500079

[10] J. Cheeger, Differentiability of Lipschitz functions on metric measure spaces. Geom. Funct. Anal. 9(1999), 428-517. http://dx.doi.org/10.1007/s000390050094

[11] M. Erbar, K. Kuwada, and K.-T. Sturm, On the equivalence of the entropic curvature-dimension condition and Bochner's inequality on metric measure spaces. Invent. Math. 201(2015), 993-1071. http://dx.doi.org/10.1007/s00222-014-0563-7

[12] F. Galaz-Garcia, M. Kell, A. Mondino, and G. Sosa, On quotients of spaces with Ricci curvature bounded below. arxiv: 1704.05428

[13] N. Gigli, Optimal maps in non branching spaces with Ricci curvature bounded from below. Geom. Funct. Anal. 22(2012), 990-999. http://dx.doi.org/10.1007/s00039-012-0176-5

[14] $\longrightarrow$ The splitting theorem in non-smooth context. 2013. arxiv:1302.5555

$[15] \longrightarrow$ An overview of the proof of the splitting theorem in spaces with non-negative Ricci curvature. Anal. Geom. Metr. Spaces 2(2014), 169-213. http://dx.doi.org/10.2478/agms-2014-0006

[16] $\longrightarrow$ On the differential structure of metric measure spaces and applications. Mem. Amer. Math. Soc. 236(2015), no. 1113. http://dx.doi.org/10.1090/memo/1113 
[17] N. Gigli and A. Mondino, A PDE approach to nonlinear potential theory in metric measure spaces. J. Math. Pures Appl. (9) 100(2013), 505-534. http://dx.doi.org/10.1016/j.matpur.2013.01.011

[18] N. Gigli, T. Rajala, and K.-T. Sturm, Optimal maps and exponentiation on finite-dimensional spaces with Ricci curvature bounded from below. J. Geom. Anal. 26(2016), 2914-2929. http://dx.doi.org/10.1007/s12220-015-9654-y

[19] N. Gigli and L. Tamanini, Second order differentiation formula on $\mathrm{RCD}^{*}(K, N)$ spaces. Atti Accad. Naz. Lincei Rend. Lincei Mat. Appl. 29(2018), no. 2, 377-386. http://dx.doi.org/10.4171/RLM/811

[20] E. Hopf, Elementare Bemerkungen über die Lösungen partieller Differentialgleichungen zweiter Ordnung vom elliptischen Typus. Sitzungsberichte Preussiche Akad. Wiss. (1927), pp. 147-152.

[21]_A remark on linear elliptic differential equations of second order. Proc. Amer. Math. Soc. 3(1952), 791-793. http://dx.doi.org/10.2307/2032182

[22] M. Kell, Transport maps, non-branching sets of geodesics and measure rigidity. Adv. Math. 320(2017), 520-573. http://dx.doi.org/10.1016/j.aim.2017.09.003

[23] S.-i. Ohta, On the measure contraction property of metric measure spaces. Comment. Math. Helv. 82(2007), 805-828. http://dx.doi.org/10.4171/CMH/110

[24] T. Rajala, Local Poincaré inequalities from stable curvature conditions on metric spaces. Calc. Var. Partial Differential Equations 44(2012), 477-494. http://dx.doi.org/10.1007/s00526-011-0442-7

[25] T. Rajala and K.-T. Sturm, Non-branching geodesics and optimal maps in strong $C D(K, \infty)$-spaces. Calc. Var. Partial Differential Equations 50(2014), 831-846. http://dx.doi.org/10.1007/s00526-013-0657-x

[26] F. Santambrogio, Optimal transport for applied mathematicians. Calculus of variations, PDEs, and modeling. In: Progress in Nonlinear Differential Equations and their Applications, 87, Birkhäuser/Springer, Cham, 2015.

[27] K.-T. Sturm, On the geometry of metric measure spaces. II. Acta Math. 196(2006), 133-177. http://dx.doi.org/10.1007/s11511-006-0003-7

[28] C. Villani, Optimal transport. Old and new. Grundlehren der Mathematischen Wissenschaften, 338, Springer-Verlag, Berlin, 2009. http://dx.doi.org/10.1007/978-3-540-71050-9

SISSA, 34136 Trieste, Italy

e-mail: ngigli@sissa.it

Hausdorff center for Mathematics, D-53115 Bonn, Germany

e-mail: crigoni@sissa.it 